\title{
An Implanted Vestibular Prosthesis Improves Spatial Orientation in Animals with Severe Vestibular Damage
}

\author{
${ }^{\circledR}$ Faisal Karmali, ${ }^{1,2 *}$ Csilla Haburcakova, ${ }^{2 *}$ Wangsong Gong, ${ }^{2}{ }^{\circledR}$ Charles C. Della Santina, ${ }^{3}$ Daniel M. Merfeld, ${ }^{4}$ \\ and Richard F. Lewis ${ }^{1,2,5}$ \\ ${ }^{1}$ Department of Otolaryngology-Head and Neck Surgery, Harvard Medical School, Boston, Massachusetts 02114, ${ }^{2}$ Jenks Vestibular Physiology \\ Laboratory, Massachusetts Eye and Ear, Boston, Massachusetts 02114, ${ }^{3}$ Department of Otolaryngology-Head and Neck Surgery, Johns Hopkins \\ School of Medicine, Baltimore, Maryland 21287, ${ }^{4}$ Department of Otolaryngology-Head and Neck Surgery, College of Medicine, The Ohio State \\ University, Columbus, Ohio 43210, and ${ }^{5}$ Department of Neurology, Harvard Medical School, Boston, Massachusetts 02114
}

Gravity is a pervasive environmental stimulus, and accurate graviception is required for optimal spatial orientation and postural stability. The primary graviceptors are the vestibular organs, which include angular velocity (semicircular canals) and linear acceleration (otolith organs) sensors. Graviception is degraded in patients with vestibular damage, resulting in spatial misperception and imbalance. Since minimal therapy is available for these patients, substantial effort has focused on developing a vestibular prosthesis or vestibular implant (VI) that reproduces information normally provided by the canals (since reproducing otolith function is very challenging technically). Prior studies demonstrated that angular eye velocity responses could be driven by canal VI-mediated angular head velocity information, but it remains unknown whether a canal VI could improve spatial perception and posture since these behaviors require accurate estimates of angular head position in space relative to gravity. Here, we tested the hypothesis that a canal VI that transduces angular head velocity and provides this information to the brain via motion-modulated electrical stimulation of canal afferent nerves could improve the perception of angular head position relative to gravity in monkeys with severe vestibular damage. Using a subjective visual vertical task, we found that normal female monkeys accurately sensed the orientation of the head relative to gravity during dynamic tilts, that this ability was degraded following bilateral vestibular damage, and improved when the canal VI was used. These results demonstrate that a canal VI can improve graviception in vestibulopathic animals, suggesting that it could reduce the disabling perceptual and postural deficits experienced by patients with severe vestibular damage.

Key words: implant; perception; prosthesis; spatial orientation; spatial orientation; vestibular nerve

Significance Statement

Patients with vestibular damage experience impaired vision, spatial perception, and balance, symptoms that could potentially respond to a vestibular implant (VI). Anatomic features facilitate semicircular canal (angular velocity) prosthetics but inhibit approaches with the otolith (linear acceleration) organs, and canal VIs that sense angular head velocity can generate compensatory eye velocity responses in vestibulopathic subjects. Can the brain use canal VI head velocity information to improve estimates of head orientation (e.g., head position relative to gravity), which is a prerequisite for accurate spatial perception and posture? Here we show that a canal VI can improve the perception of head orientation in vestibulopathic monkeys, results that are highly significant because they suggest that VIs mimicking canal function can improve spatial orientation and balance in vestibulopathic patients.

Received Aug. 16, 2020; revised Feb. 10, 2021; accepted Mar. 8, 2021.

Author contributions: F.K., C.C.D.S., D.M.M., and R.F.L. designed research; C.H., W.G., D.M.M., and R.F.L. performed research; F.K., C.H., and R.F.L. analyzed data; F.K. and R.F.L. wrote the paper.

C.C.D.S. and The Johns Hopkins University (JHU) hold royalty interests in pending and awarded patents related to vestibular implantation. C.C.D.S. holds an equity interest in and is CE0/Chief Scientific Officer of Labyrinth Devices, LLC. The terms of this arrangement are managed in accordance with JHU policies on conflicts of interest. The authors declare no other competing financial interests.

This work was funded by National Institutes of Health/National Institute on Deafness and Other Communication Disorders Grants DC-008362 and DC-013069 to R.F.L., and by a contract from MED EL Corporation to D.M.M. We thank Howard Ying for implanting the search coils, and Felipe Santos for performing the labyrinthectomies.

*F.K. and C.H. contributed equally to this work.

Correspondence should be addressed to Richard F. Lewis at richard_lewis@meei.harvard.edu.

https://doi.org/10.1523/JNEUROSCI.2204-20.2021

Copyright $\odot 2021$ the authors

\section{Introduction}

Organisms in a terrestrial environment are continuously exposed to gravity and have developed mechanisms to sense and respond to this pervasive stimulus (Lacquaniti et al., 2015; Su et al., 2017). Accurate graviception is particularly crucial for humans, who ambulate through complex environments while stabilizing their body about a small support base, because it contributes to spatial orientation (e.g., the perception of head position relative to gravity) and posture (Carriot et al., 2008; Dakin and Rosenberg, 2018). While immobile sea creatures have primitive graviceptive systems (Mayorova et al., 2018), the mammalian vestibular system evolved to sense head motion as well as gravity-the 
vestibules consist of mechanoreceptors (hair cells) embedded in the neuroepithelium of the semicircular canals (which sense angular velocity; Goldberg and Fernández, 1975) and the otolith organs (which sense gravity and linear acceleration; Grant and Best, 1987). In addition to perception and posture, vestibular information generates eye movements that stabilize images on the retina during head motion [the vestibulo-ocular reflex (VOR); Fetter, 2007].

Peripheral vestibular damage degrades gravitational and motion sensation resulting in impaired spatial orientation, posture, and vision (Strupp et al., 2016; Kattah, 2018). Because damage is typically permanent (Morrill and He, 2017), disabling, and untreatable (beyond physical therapy; Maslovara et al., 2019), considerable research has focused on replacing the damaged vestibule with a vestibular implant (VI; Gong and Merfeld, 2000, 2002; Phillips et al., 2013; Boutros et al., 2019; Guyot and Perez Fornos, 2019). VIs target patients with hair cell disorders (e.g., aminoglycoside toxicity; Guthrie, 2008) because the afferent nerves remain largely intact and electrically excitable (Hirvonen et al., 2005) and have focused on reproducing semicircular canal function. Like several recent VOR studies (Nie et al., 2013; Nguyen et al., 2016; Boutros et al., 2019), our VI senses angular head velocity in three dimensions and provides motion-modulated information to the brain using simultaneous electrical stimulation of the three canal ampullary nerves (Phillips et al., 2020). Canal VI development takes advantage of cristae anatomy-since all hair cells in a given crista are aligned, head rotation similarly affects all afferents innervating that canal (Lim and Brichta, 2016), which can be mimicked by modulating the strength of the electrical stimulation. Conversely, gravitoinertial information provided by the otolith organs has not been effectively simulated to date (see Discussion) because hair cells in the maculae are arranged in an elaborate pattern (Jaeger et al., 2008), so tilt or translation generate complex afferent modulation that cannot be mimicked by electrically stimulating otolith afferent nerves (Suzuki et al., 1969; Fernández and Goldberg, 1976).

The angular VOR is based on canal-mediated angular head velocity information driving a proportional angular eye velocity, so, unsurprisingly, a canal VI can generate qualitatively normal VOR responses in animals and humans with vestibular damage (Nie et al., 2013; Guinand et al., 2017; Boutros et al., 2019). It not known, however, whether a canal VI can improve the spatial disorientation and imbalance experienced by vestibular patients since these behaviors depend on accurate estimates of head orientation (head position relative to the gravitational reference frame of the earth). Estimates of head orientation can be modulated with surface galvanic stimulation (Niehof et al., 2019), however, and more specifically by canal-mediated velocity signals (Merfeld et al., 1999; Angelaki et al., 2004), and we previously demonstrated that tilt illusions were inducible with vertical canal electrical stimulation (Lewis et al., 2013). The Journal Club article in the Journal of Neuroscience discussing our study (Dakin et al., 2013) suggested that the next logical step was to determine whether a canal VI could improve percepts of head orientation after the vestibular system was damaged, and here we examined that specific question. We found that the accurate graviception demonstrated by normal monkeys during dynamic tilts (Lewis et al., 2008) was degraded after severe vestibular damage and improved with chronic canal VI stimulation, indicating that the canal VI improves central estimates of head orientation and therefore could mitigate the spatial disorientation and postural instability experienced by patients with severe vestibular dysfunction.

\section{Materials and Methods}

This study was approved by the Massachusetts Eye and Ear Infirmary Animal Care and Use Committee and was performed under their oversight at Massachusetts Eye and Ear Infirmary.

Experimental design. Two female rhesus monkeys (M1 and M2) were trained to perform a psychophysical task that assayed their perception of head orientation relative to gravity, using a testing paradigm (Lewis et al., 2008, 2013) derived from the subjective visual vertical (SVV) task commonly used in human subjects (Dieterich and Brandt, 2019; Cho et al., 2020). Each monkey was trained to rotate a light bar about the roll (naso-occipital) axis using a small steering wheel with the goal of aligning the light bar parallel to gravity. Once learned, each monkey performed this task in the dark without any visual orientation cues while being dynamically tilted (sinusoidal or pseudorandom trajectories) about an earth-horizontal roll axis centered between the ears with the head immobilized related to the motion platform. Eye movements generated by angular and linear motion trajectories were also measured (in complete darkness) to quantify the efficacy of the peripheral canal, VI, and otolith inputs.

SVV testing and eye movement recordings were performed in both monkeys in four vestibular states. First, the normal monkeys were tested, then bilateral vestibular hypofunction (BVH) was induced by destroying one ear with a labyrinthectomy and exposing the ear implanted with the three VI ampullary electrodes to aminoglycosides; and then the animals were tested in this BVH state. The VI was then activated and provided both a tonic (resting) rate of electrical stimulation to the three ampullary nerves of the instrumented ear and motion-modulated stimulation that was based on the angular velocity of the head in the plane of each of the three canals (chronic VI stimulation). During the months of VI stimulation, the monkeys habitually experienced motion-modulated stimulation while moving freely in their housing and were intermittently tested on the SVV task with the modulation-OFF (tonic stimulation only) and modulation-ON (tonic and motion-modulated stimulation), using the transfer function relating angular head velocity to electrical stimulation that was habitually experienced. M2 was also tested with a modulation$\mathrm{ON} \times 2$ state, which used a transfer function with twice the sensitivity of the chronically experienced stimulation.

SVV training and testing. To quantify perceived head orientation relative to gravity, we used an SVV task similar to the version we previously used (Lewis et al., 2008, 2013). Briefly, the monkey sat in a primate chair with the head immobilized and rotated a circular steering wheel mounted inside the chair. An encoder measured the wheel position and rotated the roll orientation of a light bar $(0.20 \mathrm{~m}$ long $)$, with $1^{\circ}$ of wheel rotation causing $1^{\circ}$ of bar rotation. The light bar was displayed on a monitor $0.55 \mathrm{~m}$ in front of the animal and was viewed through a circular aperture. The room and monitor were otherwise black, and the center of the monitor, aperture, and light bar were aligned with the center of the head of the animal. During training, the monkeys learned to align the light bar parallel to a reference line, which was aligned with gravity. After this was mastered, the reference line was removed, and the monkeys learned to align the light bar parallel to gravity without visual cues. During training they were tilted in roll and accurate responses were required to receive a reward. Adequate training required $\sim 6$ months, and a brief training session with the reference bar on preceded each testing session to reinforce the learned behavior.

The testing paradigm appeared identical to the training task to the animal but did not require accurate responses to receive a reward and provided no feedback about response accuracy. Using this test, we previously recapitulated in rhesus monkeys many SVV features observed in humans, such as $\mathrm{A}$ and $\mathrm{E}$ effects and tilt illusions during centrifugation (Merfeld et al., 2001; Lewis et al., 2008; Fraser et al., 2015). While the task used in our prior studies was intermittent (the light bar was extinguished after each trial and reappeared at a random orientation), the current study used a continuous SVV task (the light bar remained visible throughout the trial) to allow recordings that were uninterrupted. This task was challenging for the animals because the bar position was continuously perturbed, which required the animal to continuously rotate the wheel to cancel these perturbations. The perturbations had two components: colored noise, which was Gaussian noise with an $\mathrm{SD}$ of $3.0^{\circ}$ that was filtered using a 
first-order, low-pass filter with a cutoff of $0.03 \mathrm{~Hz}$; and an instability that caused the bar to have an angular acceleration proportional to its current velocity. Update and sampling rates were $60 \mathrm{~Hz}$.

Motion protocols and data analysis for SVV and VOR testing. For SVV testing, the primate chair was mounted on a platform rotated by a computer-controlled servomotor. The platform tilted in roll about an earth-horizontal roll (naso-occipital) axis passing through the center of the head between the ears, motion that modulates activity in the four vertical canals and the four otolith organs (primarily, the two utricles for the relatively small tilt angles we used). Sinusoidal trajectories had a fixed amplitude of $\pm 20^{\circ}$ and were performed at frequencies of $0.005,0.01$, $0.05,0.1,0.2,0.3$, and $0.4 \mathrm{~Hz}$. The order of testing was randomized with regard to frequency. The pseudorandom trajectory was a sum of sines profile that included three sinusoids at different frequencies $(0.051,0.13$, and $0.2 \mathrm{~Hz}$ ). Chair position was measured by an encoder, sampled by LabVIEW, and stored by the computer controlling the visual display. For each SVV trial, a trained operator reviewed the light bar and chair tilt angle data to identify segments in which the monkeys were not attending to the task-based on extensive observation during the training and testing trials, these periods of inattention were defined as data segments during which the animal did not turn the wheel for $>10 \mathrm{~s}$ and/ or data segments where the light bar deviated $>45^{\circ}$ from upright, and these data were excluded from further analysis. The "perceived roll tilt angle" was calculated as the difference of the head/chair tilt angle and the SVV error (angular distance of the light bar from upright; Lewis et al., 2008). The tilt gain was determined for each frequency and vestibular state and was defined as the ratio of the [amplitude of the perceptual roll tilt report]/[amplitude of the actual tilt].

In the normal animal, roll tilt modulates activity in the four vertical canals and the four otolith organs (primarily the two utricles for the small tilt angles we used, which are orientated close to the earth-horizontal plane with the head upright). We therefore measured VOR responses during roll tilt and during interaural (IA) linear translation (to measure utricular function) in each vestibular state. Specifically, we measured the angular VOR during roll tilt about an earth-horizontal axis using sinusoidal rotations $(0.5 \mathrm{~Hz}, 20 \%$ seak velocity), and the IA linear VOR along an earth-horizontal axis during sinusoidal translations $(0.7 \mathrm{~Hz}$, $44 \mathrm{~cm} / \mathrm{s}$ peak velocity). Three-dimensional eye movements were recorded in one eye using standard search coil methods and were calibrated with a gimbal, with $5 \mathrm{~V}$ equaling $20^{\circ}$ for horizontal and vertical eye movements and $15^{\circ}$ for torsional eye movements. Eye movement traces were desaccaded using standard methods (Lewis et al., 2010), and each trace was manually reviewed by an experienced user.

Instrumentation. The following four surgical procedures were performed under general anesthesia. (1) A head bolt was attached to the skull using inverted screws set in dental cement. Relative to the stereotactic zero, it was pitched forward $\sim 18^{\circ}$ so the lateral and vertical canals were close to alignment with earth horizontal and vertical, respectively, when the head was immobilized (Blanks et al., 1985). A headcap affixed to the bolt housed the sensors of the VI and stimulation electronics and connectors for the ear electrodes and eye coils. (2) Frontal and sagittal eye coils (Judge et al., 1980) were implanted in one eye under the conjunctiva. (3) Three multisite, platinum stimulation electrodes (MED-EL) were inserted into the three canals of one ear. After drilling openings into the lateral, posterior, and superior semicircular canals near the ampullae, the electrodes were inserted and their position was adjusted using a micromanipulator to maximize eye movements (generated by short pulse trains) in the direction corresponding to the plane of the stimulated canal while simultaneously minimizing eye movements corresponding to other canals or facial movements (for surgical details, see Merfeld et al., 2007; Lewis et al., 2013). After optimally positioning each electrode, the leads were sutured to the skull and then tunneled subcutaneously to the headcap. (4) After confirming postoperatively that the electrodes functioned adequately, a labyrinthectomy of the contralateral ear was performed.

Vestibular ablation. We intended to use aminoglycosides to ablate vestibular function and began with intratympanic (IT) injections into each ear using gentamicin $(26.7 \mathrm{mg} / \mathrm{ml}$; Syed et al., 2015), evaluating the effect of ablation by measuring angular and linear VOR responses. Rhesus monkeys appeared to be fairly resistant to this mode of ablation, however, as 10 bilateral IT injections in M1 only modestly reduced the VOR. We next moved to systemic aminoglycosides using intramuscular streptomycin at doses described to be ototoxic in monkeys (Igarashi et al., 1966). After two courses of streptomycin administered intramuscularly in M1, substantial VOR responses remained, although they were diminished by $\sim 40 \%$ compared with normal. To finish the ablation process in M1 (and the complete ablation process in M2, where courses of IT and intramuscular aminoglycosides were omitted), we used direct irrigation of the endolymph in the implanted canal with gentamicin solution during electrode surgery. After verifying adequate function in the implanted electrodes postoperatively, in a separate surgery the contralateral ear was destroyed with a labyrinthectomy. The outcome of this process was a complete loss of vestibular function on the nonimplanted side and severe damage in the implanted ear, which resulted in the angular and linear VOR becoming undetectable. While the damage incurred in the implanted ear was severe enough to eliminate measurable VOR responses, the persistence of minimal residual function in the implanted ear could not be excluded, but this possibility would not affect the results of the study.

Tuning of the canal electrodes. Electrode tuning was performed as previously described (Merfeld et al., 2007; Lewis et al., 2010). Briefly, eye movements were recorded while pulse trains were applied using one of the three canal electrodes. Like the implant (below), pulses used in tuning experiments were biphasic and charge balanced, with each pulse $200 \mu$ s in duration and the cathodic and anodic pulses separated by $25 \mu$ s without current. During electrode tuning, all of the different current and return sites for each electrode (30 potential combinations) were tested, using different current amplitudes for the pulses. The goal was to optimize the size of the eye movements in the plane of the canal while concurrently minimizing orthogonal eye movement responses and facial nerve activation. The electrodes were also tested simultaneously with phase-locked pulses to confirm that eye movements were a linear superposition of those produced by stimulating each canal. After electrode tuning, we found that phase-locked activation of the anterior and posterior canal electrodes (simulating the VI stimulation produced by roll tilt of the head) produced substantial in-plane torsional eye movements but very small out-of-plane vertical and horizontal eye movements (each of which was $<10 \%$ the size of the in-plane torsional eye movement response). The current pulses for each canal electrode were thereby set at $240 \mu \mathrm{A}$ (anterior), $140 \mu \mathrm{A}$ (posterior), and $300 \mu \mathrm{A}$ (lateral) for M1; and $120 \mu \mathrm{A}$ (anterior), $180 \mu \mathrm{A}$ (posterior), and $100 \mu \mathrm{A}$ (lateral) for $\mathrm{M} 2$.

Implant stimulation. Angular head velocity was transduced by a threedimensional piezoelectric angular rate sensor fixed in the head cap and positioned so that the three sensor axes aligned with the sensitive axes of the three canals, and was filtered with a first-order high-pass filter with a time constant of $10 \mathrm{~s}$ (Lewis et al., 2010). Like our earlier VI experiments studying the VOR, the transfer function relating filtered angular velocity to pulserate modulation was a hyperbolic tangent that was nearly linear over most of the physiologic range of head velocities but was saturated at high and low velocities. The linear component of the transfer function for both monkeys had a slope of 3 pulses/s (pps)/( $/ \mathrm{s})$. The motion-modulated component of the VI stimulation was superimposed on a tonic stimulation rate of $200 \mathrm{pps}$, which was well above the normal baseline firing rate of vestibular afferents ( $\sim 100$ spikes/s; Goldberg and Fernández, 1975) so it could be modulated upward or downward for ipsilateral or contralateral head rotations (Merfeld et al., 2007; Lewis et al., 2010). Once the VI was activated, this chronic motion-modulated stimulation occurred continuously (e.g., while housed) except during experiments. During experimental sessions, the VI was set to modulation-OFF and modulation-ON, and for M2, modulation-ON, with the sensitivity doubled to $6 \mathrm{pps} /(\% / \mathrm{s})$ (modulation-ON $\times 2$ ), with the order of testing randomized.

Characterizing stimulation of the vestibular end-organs. As described above (concerning electrode tuning), it is likely that some current spread beyond the targeted canal ampullary nerves to affect other canal or otolith afferents. Of particular interest in this regard is the potential effect that utricular nerve stimulation could have on tilt percepts, since the utricles respond directly to gravitational (and linear acceleration) stimuli. Current spread to utricular afferents cannot be excluded in our studysingle-unit recordings would be required to positively identify VI effects 
on utricular nerve activity, and even if they were not identified in the recorded units, modulation of a subset of utricular afferents still could not be completely excluded. For these reasons, the potential behavioral effects of utricular stimulation deserve consideration with regard to our experimental design. We summarize these behavioral effects here and explain why any potential utricular nerve stimulation should not have a substantial effect on our experimental results. (1) Unlike our prior VI perceptual study (Lewis et al., 2013), which involved tonic electrical stimulation of a vertical canal with the animal stationary, the present study examines motion-modulated stimulation during roll tilt. The important issue introduced when stimulation is time variant is the temporal relationship between actual head tilt and the perception of head tilt. Specifically, the VI sensors (like the canals over the physiologic range of head rotations) transduce angular head velocity, and the VI stimulation targets the ampullary nerve, whose afferents normally encode head velocity. If tilt percepts were because of canal stimulation, as we propose, they would be appropriately in phase with head motion during sinusoidal motion (e.g., tilt percepts would be maximum when head tilt is maximum, although head velocity is transiently zero). In contrast, the utricles sense head position relative to gravity, and their afferent firing therefore encodes angular head position, not head velocity. If tilt percepts during VI stimulation were substantially because of utricular nerve stimulation; therefore, tilt percepts would be proportional to head angular velocity. During sinusoidal motion, tilt percepts would peak when head angular velocity peaks, which is $90^{\circ}$ out of phase with the veridical response (e.g., tilt percepts would be absent when head tilt is maximum because angular velocity is zero). Our results (see below) show that tilt percepts were approximately in phase with head tilt position (not head velocity), which is inconsistent with a substantial perceptual contribution derived from utricular nerve stimulation. (2) There are no experiments that have examined tilt perception during isolated electrical stimulation or ablation of the utricular nerve - as described below, eye movements produced by utricular nerve stimulation have been recorded (Suzuki et al., 1969), but not tilt percepts. Furthermore, SVV deviations toward the damaged labyrinth or vestibular nerve have been widely described in human patients (Min et al., 2007), but none of these reports identify the effects of isolated utricular damage on tilt perception. The closest analogs in the literature are the tilt percepts produced by surface galvanic electrical stimulation in human subjects, and as discussed in detail in the study by Fitzpatrick and Day (2004) and in our prior study (Lewis et al., 2013), depolarization or hyperpolarization of the entire utricular macula or afferent nerve should not induce substantial tilt percepts, based on the anatomy of hair cell orientation in the macula. (3) Prior work using binocular eye movement recordings (Suzuki et al., 1969) showed that electrical stimulation targeting the utricular nerve produced a static torsional and vertical offset between the two eyes. We specifically examined this issue in our 2013 study (Fig. 1 in Lewis et al., 2013) using binocular eye coils and found no evidence that such a torsional or vertical offset occurred during tonic vertical canal stimulation. While we cannot recapitulate these findings in our current study since eye recordings were monocular, the data presented in our 2013 study (Lewis et al., 2013) provide strong evidence that stimulation targeting only the vertical canals is capable of inducing tilt percepts. Together, these behavioral observations provide considerable evidence that tilt percepts in our experimental preparation were primarily because of canal activation, and that while utricular afferents may have been stimulated, their contribution to tilt perception was probably small.

Experimental protocol. In each vestibular state (normal, BVH, VI stimulation), the animals were typically tested three times/week. Because of delays associated with vestibular ablation and surgical recoveries, testing in the different states was separated by substantial periods of time. For M1, the experimental schedule was as follows: (1) 33 testing sessions in the normal state over a period of 11 weeks; (2) after a delay of 142 weeks (because of repeated aminoglycoside administrations), the VI was implanted and tuned; (3) 17 weeks later, the contralateral ear was ablated; (4) 18 weeks later, testing in the BVH state began and included 28 testing sessions performed over 14 weeks; and (5) 34 weeks later, the VI was activated, and $4 \mathrm{~d}$ after activation M1 was tested 11 times over 14 weeks in the VI-stimulated state. For M2, the experimental schedule was as follows: (1) 21 testing sessions in the normal state over a period of

\section{Torsional VOR during roll tilt}

M1
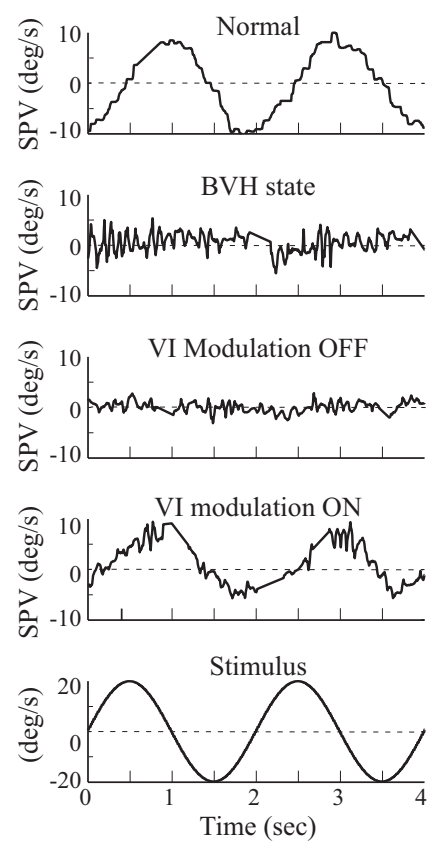

M2
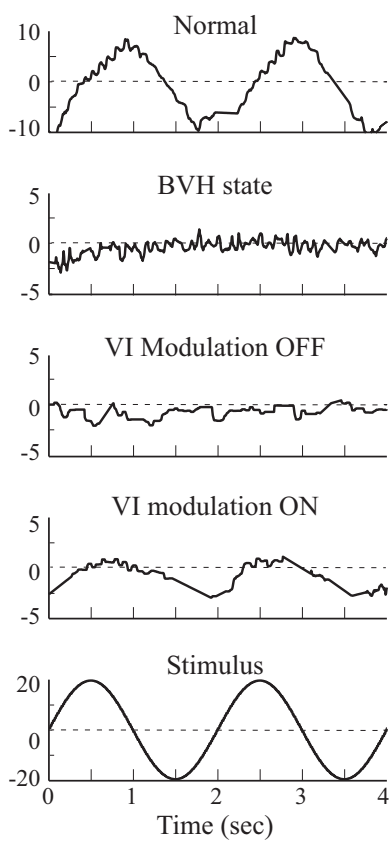

Figure 1. Torsional VOR responses during roll tilt about an earth-horizontal axis at $0.5 \mathrm{~Hz}$, $20 \%$ peak velocity, in the two monkeys and four different vestibular states: normal, BVH, semicircular canal VI modulation OFF, and VI modulation ON. The traces show desaccaded slow-phase eye velocity versus time, and the bottom trace shows angular head velocity. Eye movements were measured with implanted search coils.

8 weeks; (2) after a delay of 23 weeks, the VI was implanted and tuned; (3) 5 weeks later, the contralateral ear was ablated; (4) 13 weeks later, testing in the BVH state began and included 21 testing sessions performed over 18 weeks; and (5) 48 weeks later, the VI was activated, and $4 \mathrm{~d}$ after activation M2 was tested 37 times over 23 weeks in the VIstimulated state.

Statistical analysis. For single-frequency trials, a gain and a bias (i.e., a consistent offset) relating bar angle to chair angle were determined using a least-squares linear regression for each subject and frequency. Specifically, the following model:

$$
\hat{\bar{\theta}}_{f, s, m}=-G_{f, s, m} \cdot \bar{\theta}_{f, s, m}+B_{f, s, m},
$$

was solved to determine a gain parameter $G_{f, s, m}$ and a bias parameter $B_{f, s, m}$, where $\bar{\theta}_{f, s, m}$ is the chair tilt angle for frequency $f$, state $s$ (normal, ablated, modulation-OFF, modulation-ON), and monkey $m$, and $\hat{\bar{\theta}}_{f, s, m}$ is the corresponding perception of tilt angle. Each regression also yielded an SE for each parameter, and these were adjusted to correct for autocorrelation (hac function, Econometrics Toolbox, MATLAB 2017), since ordinary least-squares regression can underestimate SEs when data are temporally correlated. This resulted in SEs $e_{G, f, s, m}$ and $e_{B, f, s, m}$ for $G_{f, s, m}$ and $B_{f, s, m}$, respectively, which were used to compute statistical significances $p_{G, f, s, m}$ and $p_{B, f, s, m}$. Sum of sines data were also analyzed using regressions; three gain parameters and one bias parameter were determined, which related bar angle to three sinusoids at the stimulus frequencies. Specifically, the following model:

$$
\begin{gathered}
\hat{\bar{\theta}}_{s o s, s, m}=-\left(G_{s o s 1, s, m} \cdot 6^{\circ} \cdot \sin (2 \pi \cdot 0.051-t)+G_{s o s 2, s, m} \cdot 5^{\circ}\right. \\
\left.\cdot \sin (2 \pi \cdot 0.13-t)+G_{s o s 3, s, m} \cdot 6^{\circ} \cdot \sin (2 \pi \cdot 0.2-t)\right)+B_{s o s, s, m},
\end{gathered}
$$

was solved to determine gains $G_{s o s 1, s, m}, G_{s o s 2, s, m}$ and $G_{s o s 3, s, m}$, and bias $B_{s o s, s, m}$, where $\hat{\bar{\theta}}_{s o s, s, m}$ is the perception of tilt angle during sum of sines 
motion for state $s$ and monkey $m$ and $-t$ was the time vector corresponding to $\hat{\bar{\theta}}_{s o s, s, m}$. As described above, SEs and $p$ values were determined for each of the four parameters. Determining phase by adding additional terms (i.e., chair position lagged by $90^{\circ}$ for single sinusoids; cosine terms for sum of sines) did not improve fits. This was expected because the bar angle was generally in phase with chair tilt.

To compare gains for normal versus ablated, a linear regression was performed on data pooled across both states, using the model:

$$
\begin{gathered}
{\left[\begin{array}{c}
\hat{\bar{\theta}}_{f, s=\text { normal }, m} \\
\hat{\bar{\theta}}_{f, s=\text { ablated }, m}
\end{array}\right]=\left[\begin{array}{l}
-G_{f, s=\text { normal }, m} \cdot \bar{\theta}_{f, s=\text { normal }, m}+B_{f, s=\text { normal }, m} \\
-G_{f, s=\text { ablated }, m} \cdot \bar{\theta}_{f, s=\text { ablated }, m}+B_{f, s=\text { ablated }, m}
\end{array}\right]} \\
=\left[\begin{array}{c}
-G_{f, s=\text { normal }, m} \cdot \bar{\theta}_{f, s=\text { normal }, m}+B_{f, s=\text { normal }, m} \\
-\left(G_{f, s=\text { normal }, m}+\Delta G_{f, s=\text { ablated }, m}\right) \cdot \bar{\theta}_{f, s=\text { ablated }, m}+B_{f, s=\text { normal }, m}+\Delta B_{f, s=\text { ablated }, m}
\end{array}\right] \\
=-G_{f, s=\text { normal }, m} \cdot\left[\begin{array}{c}
\bar{\theta}_{f, s=\text { normal }, m} \\
\bar{\theta}_{f, s=\text { ablated }, m}
\end{array}\right]+\Delta G_{f, s=\text { ablated }, m} \cdot\left[\begin{array}{c}
0_{k x 1} \\
\bar{\theta}_{f, s=\text { ablated }, m}
\end{array}\right] \\
+B_{f, s=\text { normal }, m} \cdot\left[\begin{array}{c}
1_{k x 1} \\
1_{l x 1}
\end{array}\right]+\Delta B_{f, s=\text { ablated }, m} \cdot\left[\begin{array}{c}
0_{k x 1} \\
1_{l x 1}
\end{array}\right]
\end{gathered}
$$

which was solved to determine gain $G_{f, s=\text { normal }, m}, \Delta G_{f, s=\text { ablated, } m}$, the difference between gains for normal and ablated, and bias parameters $B_{f, s=\text { normal }, m}$ and $\Delta B_{f, s=\text { ablated }, m}$, where $k$ is the length of vector $\bar{\theta}_{f, s=\text { normal }, m}$ and $l$ is the length of vector $\bar{\theta}_{f, s=\text { ablated }, m}$. SEs $e_{G, f, s, m}$, $e_{\Delta G, f, s, m}, e_{B, f, s, m}$, and $e_{\Delta B, f, s, m}$, and corresponding statistical significances $p_{G, f, s, m}, p_{\Delta G, f, s, m}, p_{B, f, s, m}$, and $p_{\Delta B, f, s, m}$ were determined for each of the four parameters after performing the corrections described above. This approach yielded the same gain values as above, but provided the SEs and statistical significances for the differences in gains. The same approach was used to compare modulation-OFF and modulation-ON, as well as for sum of sines data. The $95 \%$ confidence intervals for these analyses are seen in Figures 4 and 5. When comparing states across multiple frequencies, Fisher's method (i.e., Fisher's combined probability test; Fisher, 1925; Mosteller and Fisher, 1948) was used to calculate a $p$ value that combined statistical significances for individual frequencies, $p_{\Delta G, f, s, m}$. Comparisons between animals and between single-sinusoid and sum of sines results were made using the nonparametric Wilcoxon signed-rank test.

\section{Results}

\section{Eye movement responses}

Roll tilt of the head about an earth-horizontal, naso-occipital axis modulates activity in the two vertical canals and the two otolith organs in each ear (primarily, the utricles for the small tilt angles we used). To assay the efficacy of these relevant vertical canal and otolith inputs in the different vestibular states, we therefore measured the torsional angular VOR produced by roll tilt of the head, and also measured the horizontal linear VOR produced by IA translation (which modulates utricular but not canal activity). Figure 1 shows the torsional roll VOR responses and demonstrates that these eye movements were elicited in the normal state (Fig. 1, top row) but were undetectable in the BVH state (Fig. 1, second row). During VI stimulation, no torsional response was evident when the modulation component of the stimulation was OFF (Fig. 1, third row) but qualitatively normal, albeit smaller responses were recorded when the animals were tested with modulation-ON (Fig. 1, fourth row). Overall, the torsional VOR eye velocity (in degrees per second) recorded with VI modulation-ON was $65 \%$ and $15 \%$ of normal in M1 and M2, respectively. The peak-to-peak amplitude of the torsional eye deviation produced by roll tilt was $5.2^{\circ}$ for $\mathrm{M} 1$ and $4.1^{\circ}$ for M2 in the normal state, zero in the ablated and VI modulation-OFF states, and $3.4^{\circ}$ for $\mathrm{M} 1$ and $1.5^{\circ}$ for M2 in the VI modulation-ON state. Roll

\section{Linear VOR during inter-aural translation}

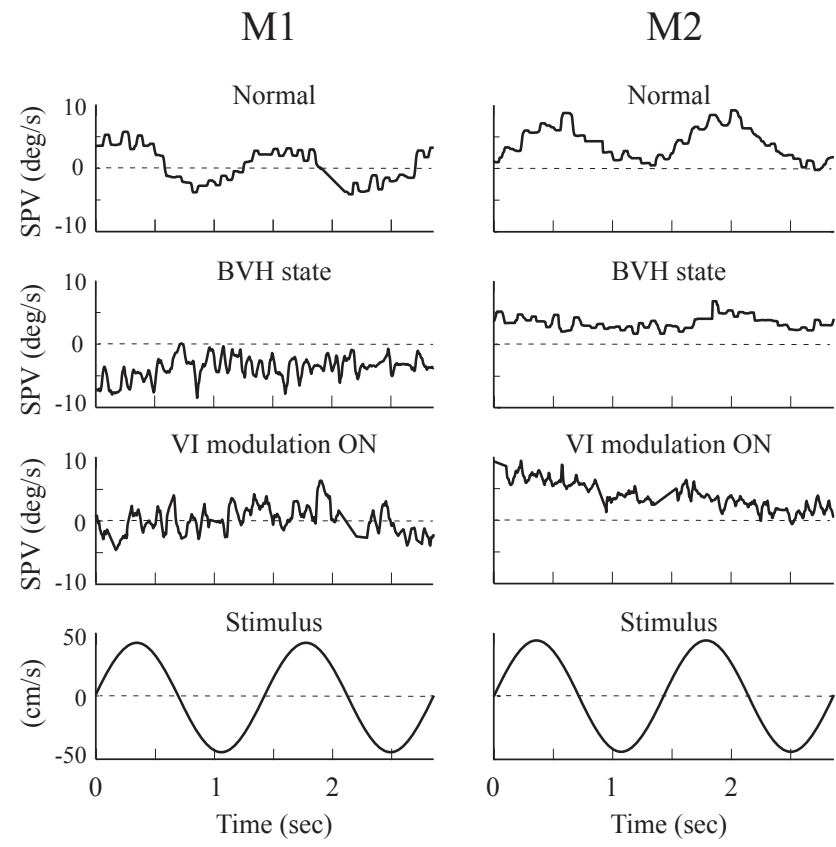

Figure 2. Horizontal eye movements in the different vestibular states induced by interaural linear acceleration along an earth-horizontal axis at $0.7 \mathrm{~Hz}$ with peak velocity of $44 \mathrm{~cm} / \mathrm{s}$.

tilt in the modulation-ON state also elicited very small vertical and horizontal eye movement responses-the former from imperfect cancellation of the vertical eye movements produced by the anterior and posterior canal electrodes and the latter because of current spread beyond the vertical canal ampullary nerves (see Materials and Methods; electrode tuning). These very small nontorsional eye movements (each of which was $<10 \%$ the magnitude of the torsional response) would not affect the roll tilt percepts recorded with the SVV task in this study but rather could potentially be associated with very small yaw or pitch percepts, neither of which was measured.

Figure 2 shows the horizontal linear IA VOR responses and demonstrates that the horizontal eye movements elicited in the normal state (Fig. 2, top row) were undetectable after the ablation procedure (indicating severe bilateral utricular damage) and remained so during VI canal stimulation. Together, these eye movement results indicate the following: (1) that function in the vestibular organs that normally modulates during roll tilt (vertical canals, otolith organs) was severely damaged by the ablative procedures, as evidenced by the undetectable angular roll tilt VOR and linear IA VOR responses in the BVH state; and (2) that motion-modulated VI stimulation of the two vertical canals of one ear provided the brain with adequate information about roll rotation to generate a compensatory torsional VOR response, albeit one that was smaller than normal and differed in size between the two monkeys.

\section{Perceptual responses}

\section{Overview}

Figure 3 shows examples of SVV responses in each of the four states, with the roll tilt angle of the head in gray and the perceived tilt angle in black, as the animals were tilted en bloc about the earth-horizontal roll axis with a sinusoidal $(0.1 \mathrm{~Hz})$ motion trajectory. In the normal state (Fig. 3, top row), both monkeys perceived roll tilt with excellent accuracy (amplitude and phase 


\section{Roll Tilt Perception}
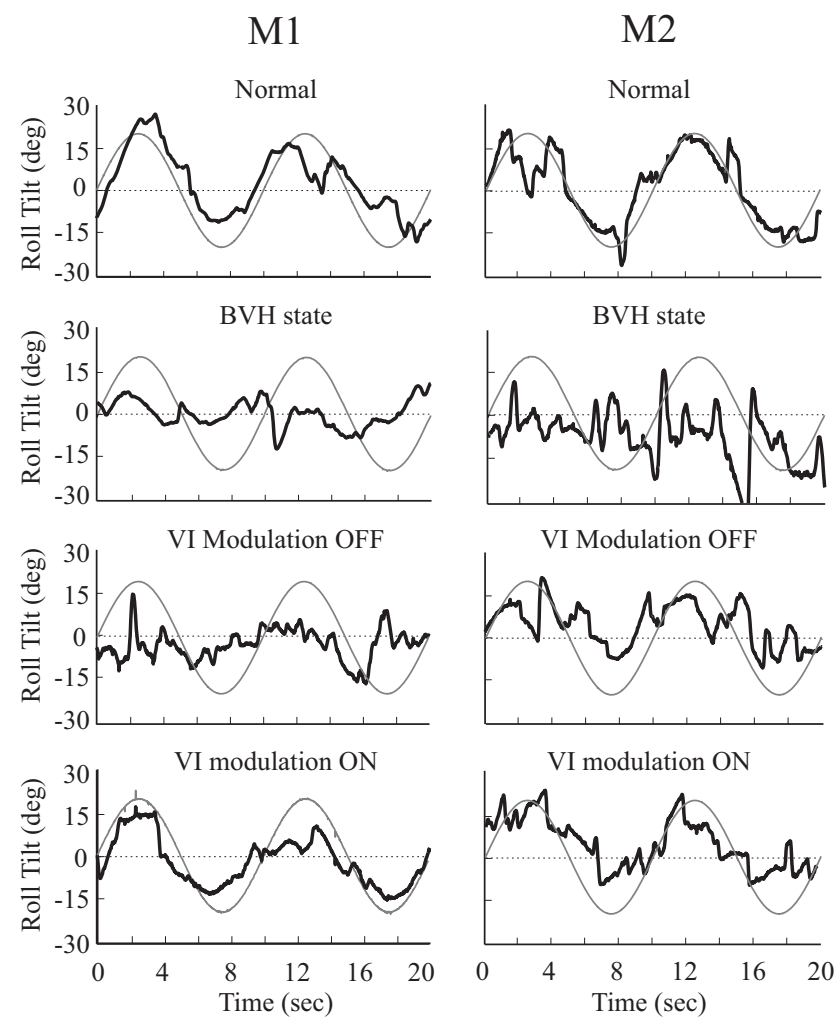

VI Modulation OFF

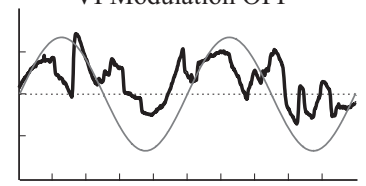

VI modulation $\mathrm{ON}$

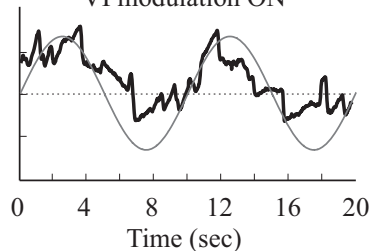

Figure 3. Tilt perception for the two monkeys in the four vestibular states. Head roll tilt (gray traces) was sinusoidal at $0.1 \mathrm{~Hz}$ with peak tilt angle of $\pm 20^{\circ}$. Perceived roll tilt (black traces) was calculated from subjective visual vertical responses, as detailed in the Materials and Methods.

of perceived tilt relative to the actual tilt); after ablation (Fig. 3, second row), their percepts of tilt decreased substantially; during VI stimulation with the modulation-OFF (Fig. 3, third row), perceived tilt remained small; and with VI modulation-ON (Fig. 3, fourth row) substantially larger perceived tilt amplitudes were recovered that were approximately in phase with the actual head tilt angle. Below we discuss the tilt values calculated in the different vestibular states, but note here (and consider in detail in the Discussion) that the specific SVV task and motion protocols almost certainly affected the amplitude of these responses. Similarly, the assumption that the tilt gain can be approximated as the linear sum of the components derived from separate graviceptive cues is implicit in the Results but is considered in detail in the Discussion.

\section{Tilt perception in normal monkeys}

Figure 4 shows the tilt gains measured in the two normal monkeys (Fig. $4 A, B$, dark gray icons) when they were tested with sinusoidal motion trajectories. The amplitude of the rotation was constant across frequencies $\left( \pm 20^{\circ}\right)$, so angular velocities and accelerations were larger for the higher frequencies (as velocity scales linearly with frequency and acceleration scales with frequency ${ }^{2}$ ). Both monkeys were tested at two mid-frequencies, 0.1 and $0.2 \mathrm{~Hz}$ (identified in bold on the $x$-axes), while testing in M1 extended to higher frequencies $(0.3-0.4 \mathrm{~Hz})$ and testing in $\mathrm{M} 2$ extended to lower frequencies $(0.005-0.05 \mathrm{~Hz})$. Together, perceptual testing in both animals covered a nearly 2 decade frequency range.

Across all frequencies, tilt gains in the normal state ranged from 0.65 to 1.0 with a mean normal tilt gain of 0.72 for $\mathrm{M} 1$ and
Tilt gains in normal and BVH states

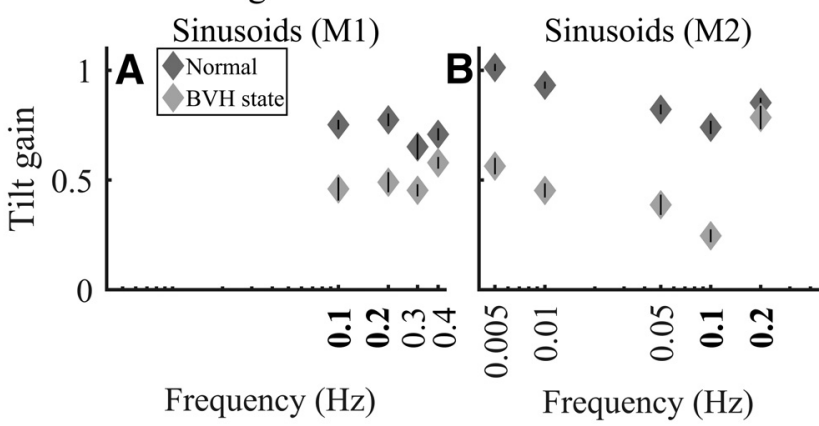

Change in tilt gain due to ablation

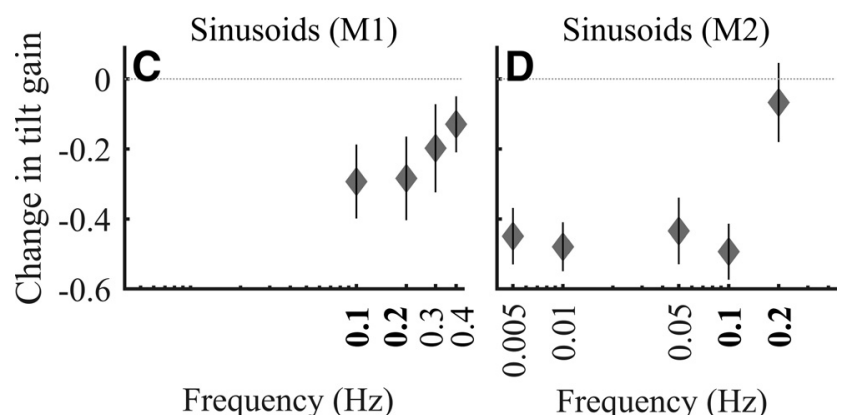

Figure 4. $\quad A, B$, Tilt gain for each monkey versus frequency for sinusoidal motion stimuli with the animals tested in the normal state (dark gray icons) and in the BVH state (light gray icons), with error bars representing $95 \%$ confidence intervals. C, D, The difference between the normal and ablated states, which approximates the vestibular contribution to tilt perception as a function of frequency. Common testing frequencies for the two monkeys were 0.1 and $0.2 \mathrm{~Hz}$ (bold, $x$-axis).

0.88 for M2. M1 gains had a flat distribution over the $0.1-0.4 \mathrm{~Hz}$ range; M2 gains trended higher at the lower frequencies (mean gain, 0.97 for 0.005 and $0.01 \mathrm{~Hz}$ ) versus higher frequencies (mean gain, 0.80 for $0.05,0.1$, and $0.2 \mathrm{~Hz}$ ). Tilt gains were similar in the two monkeys at the common frequencies $(0.1$ and $0.2 \mathrm{~Hz}$; average difference, 0.03; Wilcoxon signed-rank test, $p=1.0$ ). Of note, robust tilt perception was observed at the two very low frequencies $(0.005$ and $0.01 \mathrm{~Hz})$, which were below the cutoff frequency of the rhesus canals $(0.03 \mathrm{~Hz}$; Goldberg and Fernández, 1975). In sum, roll tilt perception in normal rhesus monkeys measured during sinusoidal motion trajectories was approximately veridical over a wide frequency range, including very low frequencies where canal modulation was minimal, indicating that noncanal (e.g., otolith, somatic, predictive) orientation cues must also contribute to tilt perception measured with these protocols.

\section{Effects of vestibular ablation on tilt perception}

Vestibular ablation induced a state of severe BVH and substantially reduced tilt gains (Fig. $4 A, B$, light gray icons, $C, D$ ). Tilt gains after vestibular ablation were between 0.4 and 0.6 in M1 and between 0.2 and 0.7 in M2. The effect of vestibular ablation was small, however, for the highest frequency tested in each monkey $(0.4 \mathrm{~Hz}$ in $\mathrm{M} 1 ; 0.2 \mathrm{~Hz}$ in M2). Across all frequencies, the vestibular contribution to tilt perception, estimated as the difference in tilt gain between the normal and BVH states, was 0.22 in M1 (31\% of the normal gain; Fisher's method, $p<0.001$ ) and 0.38 in M2 (45\% of normal; Fisher's method, $p<0.001$ ). Residual tilt percepts in the BVH state were not because of inadequate vestibular ablation since angular and linear VOR responses were absent, and therefore they appear to be nonvestibular in origin. As noted in the Discussion, nonvestibular cues 
Tilt gains in BVH state with VI modulation OFF and ON

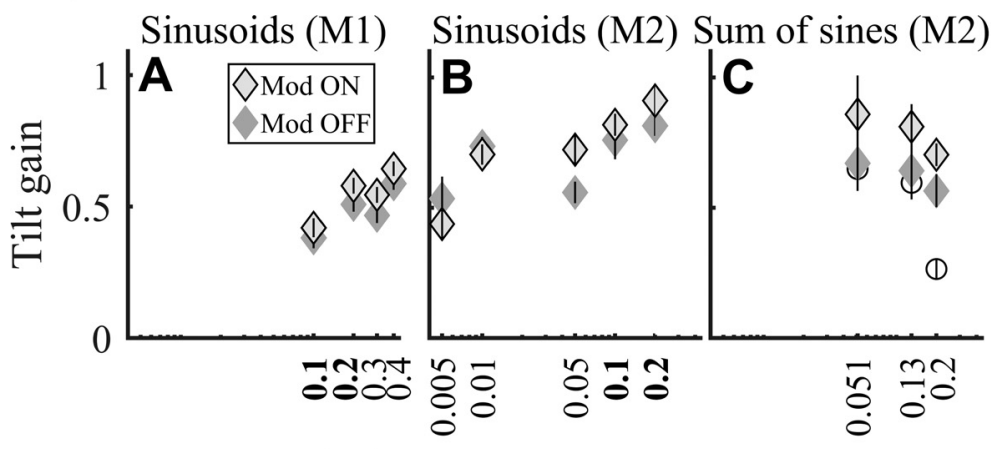

Frequency $(\mathrm{Hz})$ Frequency $(\mathrm{Hz})$ Frequency $(\mathrm{Hz})$

\section{Change in tilt gain due to VI modulation}

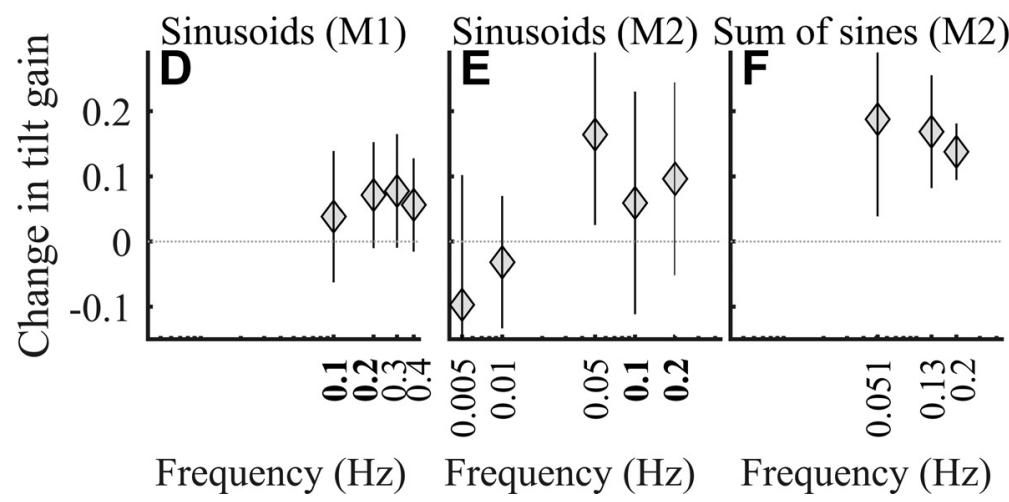

Figure 5. A-C, Tilt gains in the BVH/VI state with the motion modulation-OFF (dark gray icons and modulation-0N (light gray icons), with error bars indicating 95\% confidence intervals. $\boldsymbol{D}-\boldsymbol{F}$, The changes in tilt gain produced by motion-modulated VI stimulation, calculated as the [VI modulation ON] - [VI modulation OFF] gains for each motion trajectory. Open circles in $C$ are the sum of sines tilt gains for M2 in the BVH state without stimulation.

\section{M2 sum-of-sines trajectory}
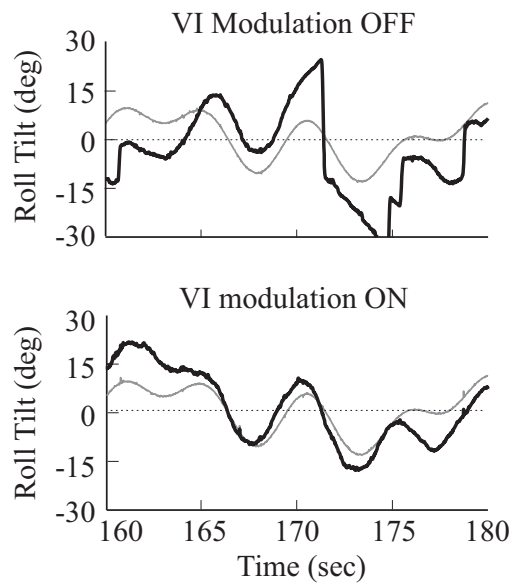

Figure 6. Sample subjective visual vertical responses during sum of sines roll tilt motions (gray traces) with black traces show the perceived roll tilt. The top panel is for the BVH modulation-OFF state, and the bottom panel is for the BVH modulation-ON state.

were probably greater at higher frequencies (rendering vestibular cues less important), which may explain the larger effect of vestibular ablation on tilt gain at lower frequencies. Together, these results show that the vestibular organs make a substantial contribution to roll tilt perception measured with our protocols, as evidenced by the large reduction in tilt gain after vestibular ablation.

Effects of prosthetic stimulation on tilt perceptionsinusoidal motion trajectories

Each monkey received chronic motion-modulated stimulation; while moving freely in their housing, VI stimulation was continuously provided to the three implanted canals. Stimulation included both a tonic stimulation rate and a superimposed motion-modulated component based on high-pass-filtered angular head velocity (cutoff frequency, $0.016 \mathrm{~Hz}$; corresponding to a $10 \mathrm{~s}$ time constant) in the plane of each canal. Figure 5 shows the modulation-OFF (dark gray) and modulation-ON (light gray) gains for each sinusoidal frequency (Fig. 5A,B) and the difference between these two values (change in tilt gain because of VI modulation; Fig. 5D,E), with each monkey tested using the same frequencies used in the normal and BVH states. M1 showed small but significant improvements in roll tilt gain with the VI modulation ON (average increase in tilt gain, 0.06; Fisher's method across all tested frequencies, $p=0.0017$; Fig. $5 A, D)$. M2 showed changes in tilt gain similar to those in $\mathrm{M} 1$ for the two frequencies where they overlapped $(0.1,0.2 \mathrm{~Hz})$ and a larger VI effect at $0.05 \mathrm{~Hz}$ (average increase in tilt gain of 0.11 ; Fisher's method across $0.05,0.1$, and $0.2 \mathrm{~Hz}$, $p=0.011$; Fig. $5 B, E$ ). As a control, we also tested the effect of VI modulation in M2 at the two very low-motion frequencies $(0.005,0.01 \mathrm{~Hz})$ used in the normal and BVH states, where pulse rate modulation was extremely small (1.9 and 3.8 pps maximum, since it scales with frequency). As expected, VI modulation did not improve tilt perception at these two very low frequencies (Fisher's method, $p=0.98$; Fig. 5E). Together, these results demonstrate that VI motion-modulated stimulation improved tilt perception in BVH animals and resulted in recovery of a substantial portion of the normal vestibular contribution to tilt perception identified with ablation-vestibular ablation inactivated the four vertical canals and the four otolith organs, and, replacing two vertical canals in one ear with VI stimulation, recovered $27 \%$ of the vestibular contribution to tilt perception in $\mathrm{M} 1$ and $32 \%$ of the vestibular contribution to tilt perception in M2.

\section{Effects of prosthetic stimulation on tilt perception-pseudoran- dom motion trajectories}

One possible reason why VI effects on tilt gain were relatively small is that tilt perception with VI modulation-OFF remained substantial (Fig. 5A,B). This finding indicates that nonvestibular cues provided considerable information about head orientation after vestibular ablation, which could render vestibular/VI contributions less essential. These nonvestibular cues are primarily somatosensory and predictive, and the latter may have been augmented by the stereotyped nature of the sinusoidal trajectories. To evaluate the role of prediction, we used a pseudorandom sum of sines trajectory in M2 that combined $0.051,0.13$, and $0.2 \mathrm{~Hz}$ frequencies (Fig. 6), which were very close to the three higher sinusoidal frequencies tested in isolation in this monkey $(0.05,0.1$, and $0.2 \mathrm{~Hz}$ ). The VI effect on gain was 0.16 (Fisher's method, $p=0.017$ ) for pseudorandom tilts (Fig. 5C,F). This was 45\% larger than the VI effect for sinusoids at these frequencies $(0.11)$, but this 
difference was not statistically significant (Wilcoxon signed-rank test, $p=0.10)$. For the two higher-frequency pairs (0.1/0.13 and $0.2 /$ $0.2 \mathrm{~Hz}$; Fig. $5 B, C$ ), the modulation-OFF tilt gains during pseudorandom motion were smaller than the gains during sinusoidal tilts, and, in conjunction, the effects of VI motion modulation on the tilt gain (Fig. $5 E, F$ ) increased at these two frequencies. Although these results were not statistically significant, they are consistent with the idea that larger VI effects on tilt perception in the BVH animals could be demonstrated if nonvestibular orientation cues were reduced (e.g., eliminating prediction with pseudorandom testing).

\section{Evidence of adaptation during VI stimulation}

The monkeys habitually experienced the VI modulation-ON state in their housing with the transfer function providing a 3 $\mathrm{pps} /(\% \mathrm{~s})$ motion modulation, but during testing $\mathrm{M} 2$ was studied in this chronic "ON $\times 1$ " state and also with the modulation sensitivity doubled $(6 \mathrm{pps} /(\% / \mathrm{s}), \mathrm{ON} \times 2)$. The rationale for testing the $\mathrm{ON} \times 2$ state was to determine whether the VI effect on tilt gain was solely dependent on the sensitivity of the motion modulation, or whether brain required a period of VI exposure in concert with the other sensorimotor cues that occur during volitional movement. Focusing on the three sinusoidal stimuli that generated significant VI motion modulation $(0.05,0.1$, and $0.2 \mathrm{~Hz}$ ), we found that tilt gains were $12.2 \%$ smaller in the $\mathrm{ON} \times$ 2 state compared with the $\mathrm{ON} \times 1$ state (Fisher's method, $p=0.031$ ), although motion-modulated sensitivity was doubled in the $\mathrm{ON} \times 2$ state. These results suggest that the brain requires exposure to VI stimulation coupled to other sensorimotor motion cues during normal voluntary movements to use the prosthetic vestibular information effectively.

\section{Discussion}

Our principal finding is that a unilateral canal VI improves the perception of head orientation in rhesus monkeys with severe peripheral vestibular damage. Below, we discuss the significance of these findings, considerations in interpretation and methodology, and an approach to translate vestibular implants into the clinic.

\section{Improves graviception}

\section{Translational implications}

BVH affects $\sim 64,000$ American adults (Ward et al., 2013), is permanent, and lacks any treatment other than physical therapy, which offers modest benefits. Since BVH patients experience spatial disorientation, imbalance, and visual blurring, it is unsurprising that their quality of life is severely diminished (Sun et al., 2014). Two therapies could potentially restore function in BVH patients when hair cell death underlies their deficit: regeneration (Burns and Stone, 2017) and prosthetics. While hair cell regeneration has not yet demonstrated meaningful behavioral effects (Sayyid et al., 2019), the canal VI can simulate the angular velocity input normally provided by the canals, as evidenced by its ability to drive a compensatory VOR (Nie et al., 2013; Guinand et al., 2017; Boutros et al., 2019). VOR recovery should improve vision in BVH patients, but mechanistically is a relatively simple eye velocity response driven by head velocity sensed by the canals/VI. Since spatial perception and balance depend on accurate estimates of angular head position relative to gravity, one major unresolved problem in vestibular prosthetics is to determine whether the brain can use VImediated angular velocity information to improve central estimates of angular head position.

Our results indicate that a canal VI can improve the ability of vestibulopathic animals to estimate angular head position, as evidenced by improved percepts of spatial (head) orientation. Moreover, the VI reproduced a substantial fraction of the normal vestibular contribution to tilt perception, the perceptual effects were similar in size to VI-mediated VOR effects (Lewis et al., 2010; Nie et al., 2013), and they paralleled the amount of vestibular information supplied by the prosthesis relative to the normal labyrinth. These salutary effects on graviception should translate into improved spatial orientation and balance in vestibulopathic patients (Peterka, 2002; Stapley et al., 2006).

\section{Mechanistic implications}

As linear accelerometers, the otolith organs cannot segregate gravity from linear acceleration, and prior work strongly implies that temporal integration of canal-mediated angular velocity information can be used to help identify gravity (Merfeld et al., 2001, 2005; Park et al., 2006). This canal integration mechanism is also supported by our prior observation that electrically activating a vertical canal alters percepts of head orientation (Lewis et al., 2013). The current study confirms that head orientation can be estimated, at least in part, by temporally integrating the off-vertical component of the angular velocity signal provided by canal afferents. While the activation of otolith afferents by current spread from intracanalicular electrodes cannot be excluded, as discussed at length in our prior study (Lewis et al., 2013) and above in the Materials and Methods, electrical stimulation of utricular afferents is not predicted to elicit substantial veridical tilt percepts. This conclusion is supported by the anatomy of the utricular macula, the oculomotor data in our 2013 study (Lewis et al., 2013), and the temporal alignment of tilt perception with the head tilt angle rather than head angular velocity.

\section{Synthesis of VI and other sensorimotor cues Central processing of VI information}

The reduction in VI-modulated tilt gain when implant sensitivity was doubled from its habitual state supports the contention that VI-mediated perceptual responses require central adaptation, presumably driven by habitual exposure to VI stimulation in concert with the other sensorimotor orientation cues associated with volitional movement. This conclusion is not dependent on a linear relationship between VI stimulation rate and tilt perception amplitude but does assume a monotonic relationship, which we demonstrated in our prior study (Lewis et al., 2013) over a 60-200 pps range of stimulation rates. In addition, the discordance between VI-mediated VOR and perceptual responses supports a nonreflexive mechanism for the influence of the implant on perception, as the torsional VOR gain was much larger for M1 but VI effects on tilt gain were equivalent in both monkeys.

A sensory conflict could occur in BVH/VI patients during head tilts since the implant generates angular velocity signals that identify a change in head orientation, but that damaged otolith function would not modulate appropriately. This mismatch could generate aberrant estimates of linear acceleration, as evidenced by linear VOR responses (Merfeld et al., 1999). In acute experiments, vertical canal stimulation did elicit these eye movements (Lewis et al., 2005), but we did not identify similar responses in ablated monkeys receiving VI stimulation. If linear VOR responses were induced by head tilts after VI activation, they presumably were suppressed by adaptation before our first experimental session.

\section{Chronic effects of vestibular ablation and VI stimulation}

Since the time frame of the study was prolonged, slow sensorimotor changes may have affected graviception as the monkeys 
progressed from normal $\rightarrow \mathrm{BVH}$ to $\rightarrow \mathrm{BVH} / \mathrm{VI}$ states. The vestibular contribution to tilt perception was estimated as the (normal - BVH) tilt gain difference, but BVH data were acquired months after normal data. Since the brain weighs sensory cues based on their reliability (Lim et al., 2017), degrading vestibular information should increase reliance on nonvestibular graviceptive cues, which would reduce the (normal - BVH) difference and possibly result in us underestimating the vestibular contribution to tilt perception. Roll tilt perception and angular VOR responses measured during the chronic VI stimulation period with modulation-OFF did not differ statistically from those in the earlier BVH state. There was no evidence, therefore, that chronic VI stimulation sensitized peripheral or central vestibular pathways in a manner that affected behavioral responses.

\section{Methodological issues and potential limitations}

As summarized above, measuring tilt perception during motion introduces several methodological factors that could affect the results. Our overall conclusion, however, is that our approach provided meaningful estimates of the vestibular, prosthetic, and nonvestibular contributions to tilt perception, and that other experimental approaches would likely produce quantitatively different but qualitatively similar results.

\section{Using the SVV to quantify graviception}

Potential visual effects on the SVV were considered in our prior VI study (Lewis et al., 2013), but, briefly, there is no definitive method quantify graviception (but see Dockheer et al., 2018) and the influence of torsional eye position on SVV responses remains unresolved. Like our prior study (Lewis et al., 2013), however, we found that SVV shifts exceeded the magnitude of torsional eye position changes and therefore cannot be purely ocular in origin.

\section{Motion paradigms-frequency and prediction}

Sinusoidal tilts had fixed amplitudes, so angular head velocity, and hence the VI modulation, scaled linearly with frequency. Nonvestibular orientation cues included trunk somatosensory inputs, generated by gravity and by inertial forces (which scaled with frequency ${ }^{2}$ ), and prediction during the sinusoidal trajectories. Canal/VI and nonvestibular tilt cues therefore increased with frequency, and one explanation for the observed VI frequency dependence is that larger nonvestibular cues reduced the need for the contributions of the implant-higher tilt frequencies increased VI-mediated tilt gains because of higher angular velocities but also appeared to suppress them because of larger nonvestibular tilt cues. These mechanisms are consistent with our results, since VI contributions were small at low frequencies $(0.005$ and $0.01 \mathrm{~Hz})$ and increased with frequency until nonvestibular tilt cues began to dominate, resulting in peak VI effect at $0.05 \mathrm{~Hz}$ and smaller VI effects at the higher frequencies. Since the VI tilt gain increased at 0.13 and $0.2 \mathrm{~Hz}$ with pseudorandom trajectories, prediction may have been an important tilt cue that lowered VI contributions to tilt perception at higher frequencies.

\section{Vestibular implant characteristics}

Our VI was a three-dimensional expansion of the device used in prior VOR experiments (Lewis et al., 2010) but used newly designed multisite electrodes to better control current pathways. While our focus was to extend this device to the perceptual realm, it is probable that VI-mediated perceptual effects could be enhanced by refining the technical characteristics of the implant and by expanding from unilateral to bilateral stimulation (Gong et al., 2008).

\section{Conclusions - translating the VI to the clinic}

Considerable work remains before the VI can be optimized as a clinical therapy. Animal studies are appropriate to investigate many questions, including characterizing the electrical stimulation parameters that transfer motion information most effectively (Davidovics et al., 2012), understanding how canal VI inputs are synthesized with other sensorimotor cues (Lewis, 2015, 2016), and defining pathologic changes induced by chronic implantation (Sun et al., 2015). The initial steps toward developing an otolith VI, while technically challenging, have also recently begun in animal studies (Hageman et al., 2020a,b). Human implantation has been initiated by three groups, and results to date indicate that acute and chronic VOR responses are elicitable in BVH patients (Guinand et al., 2017; Boutros et al., 2019; Phillips et al., 2020), that functional hearing is preserved after implantation (Boutros et al., 2019), and that vestibulospinal mechanisms are engaged by acute VI stimulation (Phillips et al., 2013; Perez Fornos et al., 2019). Future animal and human work should be performed in parallel to expedite the development of the VI, since these approaches will provide complementary information about the behavioral effects, efficacy, and safety of the VI.

\section{References}

Angelaki DE, Shaikh AG, Green AM, Dickman JD (2004) Neurons compute internal models of the physical laws of motion. Nature 430:560-564.

Blanks RH, Curthoys IS, Bennett ML, Markham CH (1985) Planar relationships of the semicircular canals in rhesus and squirrel monkeys. Brain Res 340:315-324

Boutros PJ, Schoo DP, Rahman M, Valentin NS, Chow MR, Ayiotis AI, Morris BJ, Hofner A, Rascon AM, Marx A, Deas R, Fridman GY, Davidovics NS, Ward BK, Treviño C, Bowditch SP, Roberts DC, Lane KE, Gimmon Y, Schubert MC, et al. (2019) Continuous vestibular implant stimulation partially restores eye-stabilizing reflexes. JCI Insight 4:e128397.

Burns JC, Stone JS (2017) Development and regeneration of vestibular hair cells in mammals. Semin Cell Dev Biol 65:96-105.

Carriot J, DiZio P, Nougier V (2008) Vertical frames of reference and control of body orientation. Neurophysiol Clin 38:423-437.

Cho IH, Kwon JW, Yeo SS (2020) Relations between gait characteristics and subjective visual vertical results in young adults. J Vestib Res 30:73-79.

Dakin CJ, Rosenberg A (2018) Gravity estimation and verticality perception. Handb Clin Neurol 159:43-59.

Dakin CJ, Elmore LC, Rosenberg A (2013) One step closer to a functional vestibular prosthesis. J Neurosci 33:14978-14980.

Davidovics NS, Fridman GY, Della Santina CC (2012) Co-modulation of stimulus rate and current from elevated baselines expands head motion encoding range of the vestibular prosthesis. Exp Brain Res 218:389-400.

Dieterich M, Brandt T (2019) Perception of verticality and vestibular disorders of balance and falls. Front Neurol 10:172.

Dockheer KM, Bockisch CJ, Tarnutzer AA (2018) Effects of optokinetic stimulation on verticality perception are much larger for vision-based paradigms than for vision-independent paradigms. Front Neurol 9:323.

Fernández C, Goldberg JM (1976) Physiology of peripheral neurons innervating otolith organs of the squirrel monkey. II. Directional selectivity and force-response relations. J Neurophysiol 39:985-995.

Fetter M (2007) Vestibulo-ocular reflex. Dev Ophthalmol 40:35-51.

Fisher RA (1925) Statistical methods for research workers. Edinburgh, UK: Oliver and Boyd.

Fitzpatrick RC, Day BL (2004) Probing the human vestibular system with galvanic stimulation. J Appl Physiol 96:2301-2316.

Fraser LE, Makooie B, Harris LR (2015) The subjective visual vertical and the subjective haptic vertical access different gravity estimates. PLoS One 10: e0145528.

Goldberg JM, Fernández C (1975) Responses of peripheral vestibular neurons to angular and linear accelerations in the squirrel monkey. Acta Otolaryngol 80:101-110.

Gong W, Merfeld DM (2000) Prototype neural semicircular canal prosthesis using patterned electrical stimulation. Ann Biomed Eng 28:572-581. 
Gong W, Merfeld DM (2002) System design and performance of a unilateral horizontal semicircular canal prosthesis. IEEE Trans Biomed Eng 49:175-181.

Gong W, Haburcakova C, Merfeld DM (2008) Vestibulo-ocular responses evoked via bilateral electrical stimulation of the lateral semicircular canals. IEEE Trans Biomed Eng 55:2608-2619.

Grant W, Best W (1987) Otolith-organ mechanics: lumped parameter model and dynamic response. Aviat Space Environ Med 58:970-976.

Guinand N, Van de Berg R, Cavuscens S, Ranieri M, Schneider E, Lucieer F, Kingma H, Guyot J-P, Pérez Fornos A (2017) The video head impulse test to assess the efficacy of vestibular implants in humans. Front Neurol 8:600.

Guthrie OW (2008) Aminoglycoside induced ototoxicity. Toxicology 249:91-96.

Guyot JP, Perez Fornos A (2019) Milestones in the development of a vestibular implant. Curr Opin Neurol 32:145-153.

Hageman KN, Chow MR, Roberts D, Della Santina CC (2020a) Binocular 3D otolith-ocular reflexes: responses of normal chinchillas to tilt and translation. J Neurophysiol 123:243-258.

Hageman KN, Chow MR, Roberts D, Boutros PJ, Tooker A, Lee K, Felix S, Pannu SS, Haque R, Della Santina CC (2020b) Binocular 3D otolith-ocular reflexes: responses of chinchillas to prosthetic electrical stimulation targeting the utricle and saccule. J Neurophysiol 123:259-276.

Hirvonen TP, Minor LB, Hullar TE, Carey JP (2005) Effects of intratympanic gentamicin on vestibular afferents and hair cells in the chinchilla. J Neurophysiol 93:643-655

Igarashi M, McLeod ME, Graybiel A (1966) Clinical pathological correlations in squirrel monkeys after suppression of semicircular canal function by streptomycin sulfate. Acta Otolaryngol 61 [Suppl 214]:1- 228.

Jaeger R, Kondrachuk AV, Haslwanter T (2008) The distribution of otolith polarization vectors in mammals: comparison between model predictions and single cell recordings. Hear Res 239:12-19.

Judge SJ, Richmond BJ, Chu FC (1980) Implantation of magnetic search coils for measurement of eye position: an improved method. Vision Res 20:535-538.

Kattah JC (2018) Clinical Characteristics and Etiology of Bilateral Vestibular Loss in a cohort from central Illinois. Front Neurol 9:46.

Lacquaniti F, Bosco G, Gravano S, Indovina I, La Scaleia B, Maffei V, Zago M (2015) Gravity in the brain as a reference for space and time perception. Multisens Res 28:397-426.

Lewis RF (2015) Advances in the diagnosis and treatment of vestibular disorders: psychophysics and prosthetics. J Neurosci 35:5089-5096.

Lewis RF (2016) Vestibular implants studied in animal models: clinical and scientific implications. J Neurophysiol 116:2777-2788.

Lewis RF, Haburcakova C, Gong W, Lee D, Merfeld DM (2005) Are the semicircular canals graviceptors? Soc Neurosci Abstr 31:932.935.

Lewis RF, Haburcakova C, Merfeld DM (2008) Roll tilt psychophysics in rhesus monkeys during vestibular and visual stimulation. J Neurophysiol 100:140-153.

Lewis RF, Haburcakova C, Gong W, Makary C, Merfeld DM (2010) Vestibuloocular reflex adaptation investigated with chronic motionmodulated electrical stimulation of semicircular canal afferents. J Neurophysiol 103:1066-1079.

Lewis RF, Haburcakova C, Gong W, Lee D, Merfeld D (2013) Electrical stimulation of semicircular canal afferents affects the perception of head orientation. J Neurosci 33:9530-9535.

Lim R, Brichta AM (2016) Anatomical and physiological development of the human inner ear. Hear Res 338:9-21.

Lim K, Karmali F, Nicoucar K, Merfeld DM (2017) Perceptual precision of passive body tilt is consistent with statistically optimal cue integration. J Neurophysiol 117:2037-2052.

Maslovara S, Butkovic-Soldo S, Peric M, Pajic Matic I, Sestak A (2019) Effect of vestibular rehabilitation on recovery rate and functioning improvement in patients with chronic unilateral vestibular hypofunction and bilateral vestibular hypofunction. NeuroRehabilitation 44:95-102.

Mayorova TD, Smith CL, Hammar K, Winters CA, Pivovarova NB, Aronova MA, Leapman RD, Reese TS (2018) Cells containing aragonite crystals mediate responses to gravity in Trichoplax adhaerens (Placozoa), an animal lacking neurons and synapses. PLoS One 13:e0190905.

Merfeld DM, Zupan L, Peterka RJ (1999) Humans use internal models to estimate gravity and linear acceleration. Nature 398:615-618.

Merfeld DM, Zupan LH, Gifford CA (2001) Neural processing of gravito-inertial cues in humans. II. Influence of the semicircular canals during eccentric rotation. J Neurophysiol 85:1648-1660.
Merfeld DM, Park S, Gianna-Poulin C, Black FO, Wood S (2005) Vestibular perception and action employ qualitatively different mechanisms. II. VOR and perceptual responses during combined TilteTranslation. J Neurophysiol 94:199-205.

Merfeld DM, Haburcakova C, Gong W, Lewis RF (2007) Chronic vestibuloocular reflexes evoked by a vestibular prosthesis. IEEE Trans Biomed Eng 54:1005-1015.

Min KK, Ha JS, Kim MJ, Cho CH, Cha HE, Lee JH (2007) Clinical use of subjective visual horizontal and vertical in patients of unilateral vestibular neuritis. Otol Neurotol 28:520-525.

Morrill S, He DZZ (2017) Apoptosis in inner ear sensory hair cells. J Otol 12:151-164.

Mosteller F, Fisher RA (1948) Questions and answers. Am Stat 2:30-31.

Nguyen TA, DiGiovanna J, Cavuscens S, Ranieri M, Guinand N, van de Berg R, Carpaneto J, Kingma H, Guyot JP, Micera S, Fornos AP (2016) Characterization of pulse amplitude and pulse rate modulation for a human vestibular implant during acute electrical stimulation. J Neural Eng 13:046023.

Nie K, Ling L, Bierer SM, Kaneko CR, Fuchs AF, Oxford T, Rubinstein JT, Phillips JO (2013) An experimental vestibular neural prosthesis: design and preliminary results with rhesus monkeys stimulated with modulated pulses. IEEE Trans Biomed Eng 60:1685-1692.

Niehof N, Perdreau F, Koppen M, Medendorp WP (2019) Contributions of optostatic and optokinetic cues to the perception of vertical. J Neurophysiol 122:480-489.

Park S, Gianna-Poulin C, Black FO, Wood S, Merfeld DM (2006) Roll rotation cues influence roll tilt perception assayed using a somatosensory technique. J Neurophysiol 96:486-491.

Peterka RJ (2002) Sensorimotor integration in human postural control. J Neurophysiol 88:1097-1118.

Perez Fornos A, van de Berg R, Armand S, Cavuscens S, Ranieri M, Crétallaz C, Kingma H, Guyot JP, Guinand N (2019) Cervical myogenic potentials and controlled postural responses elicited by a prototype vestibular implant. J Neurol 266:33-41.

Phillips C, Defrancisci C, Ling L, Nie K, Nowack A, Phillips JO, Rubinstein JT (2013) Postural responses to electrical stimulation of the vestibular end organs in human subjects. Exp Brain Res 229:181-195.

Phillips JO, Ling L, Nowack A, Rebollar B, Rubinstein JT (2020) Interactions between auditory and vestibular modalities during stimulation with a combined vestibular and cochlear prosthesis. Audiol Neurootol 25:96-108.

Sayyid ZN, Wang T, Chen L, Jones SM, Cheng AG (2019) Atoh1 directs regeneration and functional recovery of the mature mouse vestibular system. Cell Rep 28:312-324.e4.

Stapley PJ, Ting LH, Kuifu C, Everaert DG, Macpherson JM (2006) Bilateral vestibular loss leads to active destabilization of balance during voluntary head turns in the standing cat. J Neurophysiol 95:3783-3797.

Strupp M, Feil K, Dieterich M, Brandt T (2016) Bilateral vestibulopathy. Handb Clin Neurol 137:235-240.

Su SH, Gibbs NM, Jancewicz AL, Masson PH (2017) Molecular mechanisms of root gravitropism. Curr Biol 27:R964-R972.

Sun DQ, Ward BK, Semenov YR, Carey JP, Della Santina CC (2014) Bilateral vestibular deficiency: quality of life and economic implications. JAMA Otolaryngol Head Neck Surg 140:527-534.

Sun DQ, Lehar M, Dai C, Swarthout L, Lauer AM, Carey JP, Mitchell DE, Cullen KE, Della Santina CC (2015) Histopathologic changes of the inner ear in rhesus monkeys after intratympanic gentamicin injection and vestibular prosthesis electrode array implantation. J Assoc Res Otolaryngol 16:373-387.

Suzuki JI, Goto K, Tokumasu K, Cohen B (1969) Implantation of electrodes near individual vestibular nerve branches in mammals. Ann Otol Rhinol Laryngol 78:815-826.

Syed MI, Ilan O, Nassar J, Rutka JA (2015) Intratympanic therapy in Meniere's syndrome or disease: up to date evidence for clinical practice. Clin Otolaryngol 40:682-690.

Ward BK, Agrawal Y, Hoffman HJ, Carey JP, Della Santina CC (2013) Prevalence and impact of bilateral vestibular hypofunction: results from the 2008 US National Health Interview Survey. JAMA Otolaryngol Head Neck Surg 139:803-810. 